\title{
A POSSIBLE APPROACH TO THE OPTIMIZATION OF FLOW INJECTION ANALYSIS
}

\section{J. M. REIJN and H. POPPE*}

Laboratory for Analytical Chemistry, University of Amsterdam, Nieuwe Ach tergracht 166, 1018 WV Amsterdam (The Netherlands)

\section{W. E. VAN DER LINDEN}

Laboratory for Chemical Analysis, Twente University of Technology, P.O. Box 217, 7500 AE Enschede (The Netherlands)

(Received 21st April 1982)

\section{SUMMARY}

The theory of sample zone dispersion is well known for most cases of practical interest in flow injection analysis. This paper offers a theoretical analysis which allows for the optimal design of single-line flow systems. For various reactor types, a detailed analysis is provided in terms of physical constants, design parameters and constraints.

It is shown that, within practical constraints and using a pressure drop of less than 1 bar, it is possible to operate flow systems at 100 samples per hour, with a residence time of $100 \mathrm{~s}$ and a reagent consumption of $8 \mu \mathrm{l}$ for each determination. Further miniaturization of flow systems must rely on smaller detector volumes than those currently available, a situation not unlike that in liquid chromatography.

This paper is concerned with the performance of systems for flow injection analysis (f.i.a.). Three types of parameters of the flow system can be distinguished: physical constants which are characteristic of dilute aqueous solutions, like the molecular diffusion coefficient $D_{\mathrm{m}}$ and the viscosity; design parameters like the helix diameter in a coiled reactor or the bed diameter in a packed-bed reactor; and performance-limiting parameters (constraints) such as the maximum pressure drop over the reactor, the smallest acceptable tube diameter or the smallest detector volume that is allowed for. Equations for the optimal design of flow injection systems are presented, which show clearly when a constraint parameter actually limits the performance. The values of the constraint parameters are obtained from f.i.a. practice.

Although Růzicka and Hansen [1] have pointed out correctly the differences between f.i.a. and high-performance liquid chromatography (h.p.l.c.), the present treatment is based on optimization studies in h.p.l.c. by $\mathrm{Knox}$ et al. [2,3] and Guiochon [4]. Throughout this work, it is assumed that the elution (response) curve of the flow reactor (column) is Gaussian. It

\footnotetext{
aPresent address: Department of Bioengineering, University of Utah, Salt Lake City, UT 84112, U.S.A.
} 
was shown earlier [5] that the flow conditions must be chosen in such a way that the number of tanks to which the reactor is equivalent is greater than $50\left(P e_{L}>100\right)$ in order to satisfy this condition.

The following chromatographic definitions are used. The plate height $H$ is defined as:

$H=L\left(\sigma_{t} / m_{t}\right)^{2}$

In this definition $L$ is the length of the reactor, and $m_{\mathrm{t}}$ is the mean and $\sigma_{\mathrm{t}}^{2}$ the variance of the response curve. For small sample volumes, the mean virtually equals the residence time $t_{\mathrm{v}}$. The plate number $N$ (which in f.i.a. is known as the number of tanks) is given by

$N=L / H=\left(m_{t} / \sigma_{t}\right)^{2}$

In chromatography the plate number is related to the chromatographic resolution, whereas in f.i.a. the number of tanks is related to the maximum sampling frequency:

$f_{\max }=k_{1} / \sigma_{\mathrm{t}}$ or $N=\left(f_{\max } t_{\mathrm{v}} / k_{1}\right)^{2}$

(For convenience, all symbols are defined in Table 1.) When $f_{\max }$ is expressed in $(h)^{-1}$ and a pessimistic value for the base-width of the peak of $6 \sigma_{t}$ is taken, the numerical value of $k_{1}$ is 600. Most authors [1] take $k_{1}$ equal to 900 corresponding to a base-width of $4 \sigma_{\mathrm{t}}$. For a given reactor, the plate number is determined by the dispersion, when the injection volume is sufficiently small. The relevant equations for the flow conditions and the dispersion are summarized in Table 2 . When a characteristic length $d$ is introduced, these equations are generally valid for any reactor type. In open tubes, this length is equal to the tube diameter $d_{t}$; in packed reactors it is equal to the particle diameter $d_{p}$.

\section{TABLE 1}

List of sy mbols

\begin{tabular}{|c|c|c|c|}
\hline $\begin{array}{l}A \\
A_{c} \\
C \\
d \\
d_{h} \\
d_{p} \\
d_{t} \\
D_{m} \\
D n \\
f_{\max } \\
f_{V} \\
h \\
H \\
k_{2} \\
m_{t} \\
n\end{array}$ & $\begin{array}{l}\text { Free cross-sectional area of packed reactor } \\
\text { Constant in plate height equation of colled tube } \\
\text { Constant in plate height equation of straight tube } \\
\text { Characteristic length } \\
\text { Helix diameter (coiled tube) } \\
\text { Particle diameter } \\
\text { Tube diameter } \\
\text { Molecular diffusion coef ficient } \\
\text { Dean number } \\
\text { Maxumal sampling frequency } \\
\text { Volumetric flow rate } \\
\text { Reduced plate height } \\
\text { Plate height } \\
\text { Proportionality factor in sampling trequency } \\
\text { Reactor length } \\
\text { Mean of response curve (in time units) } \\
\text { Number of tanks }\end{array}$ & $\begin{array}{l}N \\
\Delta P \\
P e_{L} \\
R e \\
S c \\
(U\rangle \\
v \\
\alpha \\
\epsilon \\
\eta \\
k \\
\lambda \\
\nu \\
p \\
\sigma_{t}^{2} \\
\sigma_{v}^{0} \\
\phi\end{array}$ & $\begin{array}{l}\text { Plate number } \\
\text { Pressure drop over the reactor } \\
\text { Peclet number } \\
\text { Reynolds number } \\
\text { Schmidt number } \\
\text { Linear velocity of fluid } \\
\text { Reduced velocity } \\
\text { Ratio of sample volume and reactor volume } \\
\text { Vold fraction of packed reactor } \\
\text { Dynamic viscosity } \\
\text { Peak wid th reduction coefficient } \\
\text { Aspect ratio of coiled tube ( } d_{h} / d_{t} \text { ) } \\
\text { Kinematic viscosity } \\
\text { Ratio of tube diameter and particle diameter } \\
\text { Variance of response curve (in time units) } \\
\text { Variance of response curve (in volume units) } \\
\text { Column resistance factor }\end{array}$ \\
\hline
\end{tabular}




\section{TABLE 2}

General flow equations

\begin{tabular}{lllllll}
\hline$\langle v\rangle$ & $=d^{2} \Delta P / \phi \eta L$ & $(\mathrm{a})$ & $L$ & $=N h d$ & (e) \\
$t_{\mathrm{v}}$ & $=L /\langle v\rangle=N^{2} h^{2} \phi \eta / \Delta P$ & (b) & $d^{2}=N h v \phi \eta D_{\mathrm{m}} / \Delta P$ & (f) \\
$v$ & $=\left\langle(v) d / D_{\mathrm{m}}\right.$ & (c) & $\sigma_{\mathrm{v}}^{0}=L A N^{-1 / 2}$ & (g) \\
$h$ & $=f(v)$ & (d) & & & \\
\hline
\end{tabular}

For dilute aqueous solutions, $\eta=10^{-3} \mathrm{~N} \mathrm{~s} \mathrm{~m}^{-2}$ and $D_{\mathrm{m}}=10^{-9} \mathrm{~m}^{2} \mathrm{~s}^{-1}$.

The first equation in Table 2, Eqn. (a), relates the mean fluid velocity $\langle v\rangle$ and the pressure drop over the reactor $\Delta P$. The column resistance factor $\phi$ depends on the type of reactor. The dynamic viscosity, $\eta$, for dilute aqueous solutions has the numerical value $10^{-3}\left(\mathrm{~N} \mathrm{~s} \mathrm{~m}^{-2}\right)$. The residence time (in h.p.l.c. the elution time for a component without retention) is given by Eqn. (b). It is convenient to use dimensionless quantities for the mean fluid velocity and for the plate height. The reduced velocity is defined in Eqn. (c) and can also be written as $v=R e S c$. For dilute aqueous solutions, the kinematic viscosity, $\nu$, is $10^{-6}\left(\mathrm{~m}^{2} \mathrm{~s}^{-1}\right)$ and the molecular diffusion coefficient can be taken as $10^{-9}\left(\mathrm{~m}^{2} \mathrm{~s}^{-1}\right)$. Therefore the Schmidt number, $S c$, equals 1000. The Reynolds number, $R e$, is based on the appropriate characteristic length: $R e=\langle v\rangle d / \nu$. The reduced plate height $h$ is defined as $h=H / d$. The reduced plate height is a function of the reduced velocity and the geometry of the reactor, whereas in chromatography often other contributions (e.g., mass transport between mobile and stationary phase) are also encountered. As there is no retention of the solute in f.i.a., the plate-height equations are simpler than those in h.p.l.c. The reactor length $L$ is given by Eqn. (e). The correct value for the characteristic length $d$ is given by Eqn. (f). In order to avoid additional peak broadening in the detector [6], the detector volume should not exceed one half of the volume variance of the peak $\sigma_{\mathrm{V}}^{0}$. This parameter, which is not in common use in f.i.a., is defined as

$\sigma_{\mathrm{V}}^{0}=\langle v\rangle A \sigma_{\mathrm{t}}=f_{\mathrm{v}} \sigma_{\mathrm{t}}$

where $A$ is the free cross-area of the reactor and $f_{\mathrm{v}}$ is the volumetric flow rate (in $\mathrm{m}^{3} \mathrm{~s}^{-1}$ ). It should be noted that the parameter $4 \sigma_{\mathrm{v}}^{0}$ can be taken as an (optimistic) quantitative measure of reagent consumption per analysis. For open tubes and single bead string reactors (SBSR) [6], the free crossarea is entirely determined by $d$. For packed reactors with more particles per tube diameter, $A$ can be adjusted by variation of the tube diameter. The equations in Table 2 are completely general, but not yet in a form suitable for calculations. Therefore more detailed relationships for different reactor types are presented in later tables.

\section{OPTIMIZATION IN FLOW INJECTION ANALYSIS}

The main goal of h.p.l.c. optimization studies is to minimize the separation time with a given plate number. In f.i.a. the plate number plays no role 
on its own, because separation is not intended. The flow reactor has two functions: mixing of sample and reagent and providing a delay time for conversion of the analyte. From Eqn. (3), it can be seen that the sampling frequency is inversely proportional to the residence time: $f_{\max }=k_{1} t_{\mathrm{v}}^{-1} N^{1 / 2}$. Therefore, in the case of chemical conversions, the optimization objective can be formulated as making $N$ maximal, which is equivalent to making $\sigma_{\mathrm{t}}$ minimal, both at a fixed residence time, the value of which is dictated by the level of conversion which is aimed at. In the present treatment, the residence time is considered as a design parameter, the value of which is determined by the chemistry, temperature and concentration levels both of the analyte and the reagent solutions.

Inspection of plate-height equations reveals that in theory it is possible to obtain any plate number for instance by a decrease in characteristic length $d$. It is obvious that this approach leads to values of other experimental parameters, such as pressure drop and tube diameter, which are inacceptable from a practical, financial, measuring or other point of view. In the optimization procedure, one, more or all of these parameters may limit the performance of the flow system. It is emphasized here that the objective of maximizing the sampling frequency is not the only objective that can be optimized in a f.i.a. system. For instance, the object may be to minimize the reagent consumption per analysis or to minimize errors in the determination. In such cases, the sampling frequency can be treated as a constraint parameter which may not fall below a certain minimum value.

The intuitive approach to the objective of maximizing the sampling frequency is overall miniaturization of the system. Then at least two constraints are encountered: the pressure drop increases and the volume variance of the peak decreases, leading to impossibly small detector volumes. Intuition predicts that the optimal performance is expected at the highest available pressure drop and the lowest detector volume. In the following sections a detailed analysis is presented for different reactor types.

\section{Straight open tubes}

The relevant equations for straight open tubes are summarized in Table 3. The column resistance factor is 32 in this case. The plate-height equation is the familiar Aris-Taylor equation, which is valid [5] for $N>30$ or when $t_{\mathrm{v}} D_{\mathrm{m}} / d^{2}>0.07$. An attractive feature of Eqn. (i) seems to be the occurrence of a minimum for $h$. However this minimum $h=1 / 12^{1 / 2}$ at $v=(192)^{1 / 2}$ is

\section{TABLE 3}

Equations for straight open tubes

\begin{tabular}{lll}
\hline$\phi$ & $=32$ & $(\mathrm{~h})$ \\
$h$ & $=2 / v+C v($ in f.i.a., $C=1 / 96)$ & $(\mathrm{i})$ \\
$h$ & $=C v(\operatorname{large} v)$ & $(\mathrm{j})$ \\
$d^{2}$ & $=N h^{2} \phi \eta D_{\mathrm{m}} / C \Delta P$ & $(\mathrm{k})$ \\
$4 \sigma_{\mathrm{V}}^{0}$ & $=\pi d^{3} h N^{1}$
\end{tabular}


not of practical interest. In practical f.i.a. applications, high reduced velocities are used. In that case, the plate-height equation can be simplified. The correct tube diameter in this case is given by Eqn. (k). For an open tube, $A=\pi d^{2} / 4$ and the volume variance $\sigma_{\mathrm{V}}^{0}$ is given by Eqn. (1). In further derivations, the parameter $\left(4 \sigma_{\mathrm{v}}^{0}\right)$ will be used as a quantitative measure for the reagent consumption per analysis.

Following the analysis by Knox and Gilbert [3], equations for $d, h, l$ and $N$ can be derived which give each of these quantities as a function of the physical constants, the constraint parameters and (optionally) the design parameters. When $h$ is eliminated between Eqns. (k) and (l) the tube diameter is obtained:

$d=\left(\phi \eta D_{\mathrm{m}} / \pi^{2} \Delta P C\right)^{1 / 8}\left(4 \sigma_{\mathrm{V}}^{0}\right)^{1 / 4}$

From this equation the corresponding value for the reduced plate height is:

$h=\left(C^{3} \Delta P / \pi^{2} \phi \eta D_{\mathrm{m}}^{3}\right)^{1 / 4}\left(t_{\mathrm{v}}\right)^{-1 / 2}\left(4 \sigma_{\mathrm{v}}^{0}\right)^{1 / 2}$

From Eqn. (b), $N$ is given by

$N=\left(\pi^{2} D_{\mathrm{m}}{ }^{3} \Delta P / C^{3} \phi \eta\right)^{1 / 4}\left(t_{\mathrm{v}}\right)\left(4 \sigma_{\mathrm{v}}^{0}\right)^{-1 / 2}$

The reactor length is found from Eqn. (e):

$L=\left(D_{\mathrm{m}} \Delta P^{3} / \pi^{2} C \phi^{3} \eta^{3}\right)^{1 / 8}\left(t_{\mathrm{v}}\right)^{1 / 2}\left(4 \sigma_{\mathrm{v}}^{0}\right)^{1 / 4}$

The maximum sampling frequency is found from Eqns. (3) and (7):

$f_{\max }=k_{1}\left(t_{\mathrm{v}}\right)^{-1 / 2}\left(\pi^{2} D_{\mathrm{m}} \Delta P / C^{3} \phi \eta\right)^{1 / 8}\left(4 \sigma_{\mathrm{v}}^{0}\right)^{-1 / 4}$

From these design equations, important conclusions can be drawn. First, a high sampling frequency is favored by a small detector volume and also by high pressure drops. This supports the arguments for miniaturization, but the detector volume will soon be the constraint. For example, at a pressure drop of $1 \mathrm{bar}$ the tube diameter is $145 \mu \mathrm{m}$ for a detector volume of $1 \mu \mathrm{l}$, and it is $82 \mu \mathrm{m}$ for a detector volume of $0.1 \mu \mathrm{l}$. The sampling frequency for the latter case is twice the sampling frequency for the larger detector. Secondly, short residence times allow for high sampling frequencies. A constraint is the requirement $N>30$. Thirdly, as can be seen from Eqn. (9), high pressure drops are advantageous, although the sampling frequency is rather weakly dependent on the pressure drop. In f.i.a., the pressure drop should not exceed 1 bar $\left(10^{5} \mathrm{~N} \mathrm{~m}^{-2}\right)$. However, in chromatography when the detector volume and the plate number are fixed, the only way to decrease analysis time is to increase the pressure drop as can be seen from Eqn. (9). Note that in this case a constraint can also be posed by the tube diameter (Eqn. 5).

\section{Coiled tubes}

In helically coiled tubes, both the pressure drop and the dispersion are influenced by the secondary flow phenomenon (Tijssen [7]). Equations for coiled tubes are given in Table 4 . The Reynolds number is replaced by the Dean number $D n\left(=\operatorname{Re} \lambda^{1 / 2} ; \lambda\right.$ is the ratio of the helix diameter and the tube 


\section{TABLE 4}

Equations for coiled tubes

\begin{tabular}{llll}
\hline$\phi$ & $=32[1+f(D n)]$ & & (m) \\
$h$ & $=C v \kappa(v)$ & (large $v)$ & (n) \\
$\kappa$ & $=5.6 S c^{1 / 3} \lambda^{1 / 3} v^{-2 / 3}$ & $($ large $v)$ & (o) \\
$h$ & $=A_{c} d^{-1 / 3} v^{1 / 3}$ & & (p) \\
$A_{c}=5.6 d_{\mathrm{h}}^{1 / 3} S c^{1 / 3} C$ & & (q) \\
$d$ & $=N h^{4} \phi D_{\mathrm{m}} / \Delta P A_{\mathrm{c}}^{3}$ & (r) \\
\hline
\end{tabular}

diameter). For values of $D n>100$, the pressure drop is not different from its value in a straight tube at the same flow velocity $(f(D n) \rightarrow 0$ in Eqn. $m)$. The effect of the secondary flow on the reduced plate height can be described by a peak-width reduction coefficient $k$ (Eqn. o). An empirical correlation for $\kappa$ as a function of $D n S c^{1 / 2}$ has been presented by Van den Berg et al. [8]. In the region $12.5<D n S c^{1 / 2}<200, \kappa$ is given by $\kappa=5.6(D n$ $\left.S c^{1 / 2}\right)^{-067}$.

For lower values of the velocity, no secondary flow phenomena are observed in the dispersion. For values of $D n S c^{1 / 2}$ above 200, no reliable correlations for the dispersion are available as yet. Tijssen's correlations are probably too optimistic. Therefore, in this treatment, it will be assumed that only coils in the region described by the correlation of Van den Berg et al. [8] are taken into consideration. The results for lower velocities are presented above in the section on straight tubes.

A general strategy for the optimization of the coiled tubes is suggested by Eqn. (n). When $C$ is replaced by $C \kappa(v)$ in Eqns. (5-9), then a trial-and-error procedure enables the design equations to be solved. By a little rearrangement, Eqns. $(o-r)$ are the starting point for the design equations. In this treatment, the helix diameter $d_{\mathrm{h}}$ is considered to have a given value (e.g., $5 \mathrm{~mm}$ ). Proceeding in an entirely analogous way as in the previous section, the following equations for $d, h, N, L$ and $f_{\max }$ are obtained:

$$
\begin{aligned}
& d=\left(\phi^{2} \eta^{2} D_{\mathrm{m}} / \pi^{6} A_{\mathrm{c}}^{3} \Delta P^{2}\right)^{1 / 19}\left(t_{\mathrm{v}}\right)^{-1 / 19}\left(4 \sigma_{\mathrm{v}}^{0}\right)^{6 / 19} \\
& h=\left(A_{\mathrm{c}}^{36} \Delta P^{5} / \phi^{5} \eta^{5} \pi^{4} D_{\mathrm{m}}^{12}\right)^{1 / 38}\left(t_{\mathrm{v}}\right)^{-7 / 38}\left(4 \sigma_{\mathrm{v}}^{0}\right)^{2 / 19} \\
& N=\left(\pi^{2} D_{\mathrm{m}}^{6} \Delta P^{7} / A_{\mathrm{c}}^{18} \phi^{7} \eta^{7}\right)^{1 / 19}\left(t_{\mathrm{v}}\right)^{13 / 19}\left(4 \sigma_{\mathrm{v}}^{0}\right)^{-2 / 19} \\
& L=\left(D_{\mathrm{m}}^{2} \Delta P^{15} / A_{\mathrm{c}}^{6} \phi^{15} \eta^{15} \pi^{12}\right)^{1 / 38}\left(t_{\mathrm{v}}\right)^{17 / 38}\left(4 \sigma_{\mathrm{v}}^{0}\right)^{6 / 19} \\
& f_{\max }=k_{1}\left(t_{\mathrm{v}}\right)^{-25 / 38}\left(A_{\mathrm{c}}^{18} \eta^{7} \phi^{7} / \pi^{2} D_{\mathrm{m}}^{6} \Delta P^{7}\right)^{-1 / 38}\left(4 \sigma_{\mathrm{v}}^{0}\right)^{-1 / 19}
\end{aligned}
$$

When Eqns. (10-14) are compared with their counterparts for straight tubes, it is seen that the optimal tube diameter is now a function of the residence time $t_{\mathrm{v}}$. In practice, when only a limited number of tube diameters is available, the use of a non-optimal tube diameter will lead to a slightly increased reagent consumption or loss of maximum sampling frequency. 


\section{Packed reactors}

To a first approximation, the general flow equation (a) (Table 2) for the pressure drop remains valid in the case of packed reactors. Values for the column resistance factor $\phi$ are given by Knox and Gilbert [3] as $\phi=500$, by Guiochon [4] as $\phi=500-1000$ and by Van den Berg et al. [8] as $\phi=800$. In this section the value $\phi=1000$ will be used. The product of constants $\eta$ and $\phi$ then has the numerical value 1 . The equations for packed reactors are summarized in Table 5. A packed reactor can be operated at the minimum of the $h-v$ curve, because the volume variance of the peak can be adjusted by adjustment of the reactor diameter $d_{c}$ (Eqn.w). The parameter $\epsilon$ is the void fraction of the packed reactor, which has the value 0.4 for a properly packed reactor. The minimum of the $h-v$ curve is found as $h=2$ at $v=4$ (Knox and Gilbert [3]). The design equations now simplify considerably, although it should be kept in mind that a constraint parameter in the particle diameter $d_{\mathrm{p}}$ has been introduced deliberately here. This parameter can no longer be chosen freely, because it was decided to work at the minimum in the $h-v$ curve.

The general time equation (b) is simplified to Eqn. (u), yielding the plate number $N$ as $N=\left(t_{v} \Delta P / 4\right)^{1 / 2}$. The corresponding sampling frequency is given by

$f_{\max }=k_{1}\left(t_{\mathrm{v}}\right)^{-3 / 4}(\Delta P / 4)^{1 / 4}$

The correct particle diameter is given by (Eqn. v)

$d=\left(16 t_{\mathrm{v}} / \Delta P\right)^{1 / 4}\left(D_{\mathrm{m}}\right)^{1 / 2}$

The reactor length and the reagent consumption (from Eqn. w) are then

$L=\left(16 t_{\mathrm{V}}^{3} \Delta P D_{\mathrm{m}}^{2}\right)^{1 / 4}$

$\left(4 \sigma_{\mathrm{v}}^{0}\right)=\left(\pi \epsilon d_{\mathrm{c}}^{2}\right)\left(2 D_{\mathrm{m}} t_{\mathrm{v}}\right)^{1 / 2}$

Two conclusions can be drawn from these design equations. First, when the pressure drop is made as high as possible in order to maximize the sampling frequency, the particle diameter will be a constraint, as shown by the fact that at 1 bar the particle diameters range from 3.5 to $20 \mu \mathrm{m}$ when the residence time varies from 1 to $1000 \mathrm{~s}$. Secondly, the reagent consumption equation (18) leaves two alternatives: either the reagent consumption may be kept constant for different residence times (case $D^{\prime}$ ) so that the bed diameter must be optimized; or (case D) it can be decided to fix the bed

\section{TABLE 5}

Equations for packed reactors

\begin{tabular}{lll}
\hline$\phi$ & $=1000 ; \phi \eta=1$ & $(\mathrm{~s})$ \\
$h$ & $=2($ at the optimum;v $=4)$ & $(\mathrm{t})$ \\
$t_{\mathrm{v}}$ & $=4 N^{2} / \Delta P$ & $(\mathrm{u})$ \\
$d^{2}$ & $=8 N D_{\mathrm{m}} / \Delta P$ & $(\mathrm{v})$ \\
$4 \sigma_{\mathrm{V}}$ & $=\pi \epsilon d_{\mathrm{c}}^{2} 2 d N^{1 / 2}$ & $(\mathrm{w})$ \\
\hline
\end{tabular}


diameter $d_{c}$, so that the reagent consumption is a function of the residence time.

\section{Single bead string reactor}

For the SBSR, the pressure drop relation (a) remains approximately valid, as experiments indicated. The column resistance factor was evaluated as approximately 500 . Therefore, $\phi \eta$ is taken as 0.5 here. No rigorous plateheight equation for the SBSR is known yet. For the particular reactor described by Reijn et al. [5] the plate height $h$ was found to be $\approx 10$ for velocities from 3000 to 30000 . Later experiments confirmed this result for the same reactor geometry $\left(d_{\mathrm{p}}=0.6 \mathrm{~mm} ; d_{\mathrm{c}}=0.75 \mathrm{~mm}\right)$. This time $h=8$ was found; the latter value of $h$ will be taken here for the further deduction.

The equations for the SBSR are summarized in Table 6 . In a similar way as before, design equations are obtained for the plate number and sampling frequency:

$$
\begin{aligned}
& N=\left(\Delta P t_{\mathrm{v}} / 32\right)^{1 / 2} \\
& f_{\max }=k_{1}\left(t_{\mathrm{v}}\right)^{-3 / 4}(\Delta P / 32)^{1 / 4}
\end{aligned}
$$

In contrast to the preceding case of the packed reactor operated at the optimum flow conditions, no constraints on the characteristic length $d$ are imposed by the flow conditions for a SBSR, because the flow velocity can be varied over a wide range (Eqn. $z$ ).

However, the reagent consumption contains the characteristic length as a parameter. When the free cross-area for a SBSR is calculated as $\left(\pi \epsilon \rho^{2} d^{2}\right)$ and the term $\left(\pi \epsilon \rho^{2}\right)$ is given the numerical value 2 , then

$4 \sigma_{\mathrm{V}}^{0}=16 d^{3} N^{1 / 2}=16 d^{3}\left(\Delta P t_{\mathrm{v}} / 32\right)^{1 / 4}$

The role of the particle diameter in the SBSR can be compared with the role of the column diameter of the packed reactor.

From these design equations, the following conclusions can be drawn. First, the reactor should be operated at the highest possible pressure drop to obtain the maximal sampling frequency. Secondly, if operation with the reagent consumption as a constraint is desired (at the lowest acceptable value allowed by the detector volume), the particle diameter becomes a function of the residence time. This also influences the length of the reactor as $L=8 \mathrm{Nd}$. Finally, the reactor can be operated with a constant characteristic length, but the reagent consumption then becomes a function of the residence time.

\section{TABLE 6}

Equations for the SBSR

\begin{tabular}{lll}
\hline$\phi$ & $=500 ; \phi \eta=1 / 2$ & $(\mathrm{x})$ \\
$t_{\mathrm{v}}$ & $=32 N^{2} / \Delta P$ & $(\mathrm{y})$ \\
$d^{2}$ & $=4 N v D_{\mathrm{m}} / \Delta P$ & (z)
\end{tabular}




\section{DISCUSSION}

The design equations presented above allow a comparison of the performance between the four reactor types considered. Some graphical illustrations are now given for the sake of clarity. As pointed out above, the performance of all reactor types with respect to sampling frequency is optimal at the highest pressure drop. This parameter is fixed at 1 bar. Figure 1 presents the results for open tubes (A straight, B coiled reactor) where an additional constraint is formed by the detector volume. This was chosen as $1 \mu \mathrm{l}\left(4 \sigma_{\mathrm{V}}^{0}\right.$ is then $\left.8 \mu \mathrm{l}\right)$; yet another constraint for the open tubes is the condition for validity of the Taylor theory $N>30$, or in the present examples, $t>6$ (s). The packed reactors, i.e., the SBSR and the packed reactor operated at the optimum, are not subject to the detector volume constraint directly.

Figures 2 and 3 present the dimensions of the design. The characteristic length $d$ in Fig. 2 corresponds to the tube diameter in cases $\mathrm{A}$ and $\mathrm{B}$ and to the particle diameter in cases $C$ and $D$. The line $C^{\prime}$ gives the required particle diameter for the SBSR operated at a constant reagent consumption; line $\mathrm{D}^{\prime}$ gives the bed diameter for the packed reactor in the same case.

The reagent consumption per analysis is given in Fig. 4. This parameter was taken as a constraint in cases $\mathrm{A}, \mathrm{B}, \mathrm{C}^{\prime}, \mathrm{D}^{\prime}$. It is seen that the design corresponding to $\mathrm{D}$ for residence times lower than $30 \mathrm{~s}$ is inacceptable from

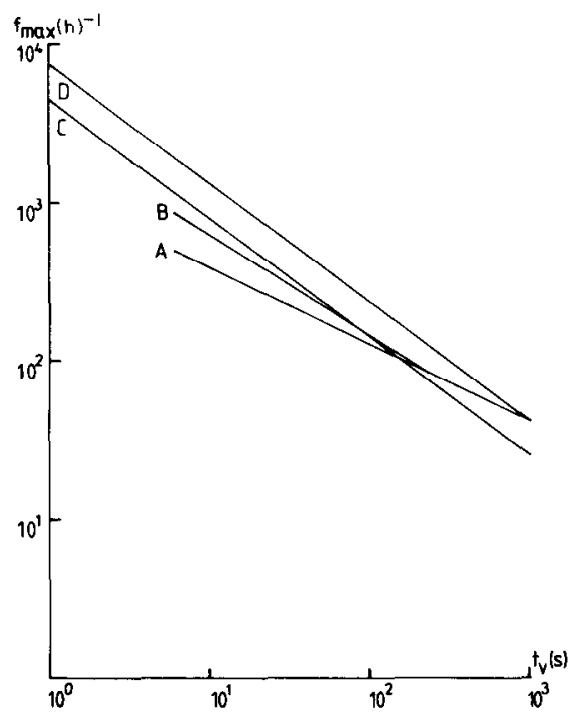

Fig. 1. Sampling frequency as a function of the residence time $t_{\mathbf{v}}$. Pressure drop, $1 \mathrm{bar}$; reagent consumption, $8 \mu 1$ for the open tubes. (A) Straight open tube; (B) coiled tube; (C) SBSR; (D) packed reactor operated at optimal conditions. Further design information is provided in Figs. 2 and 3. 

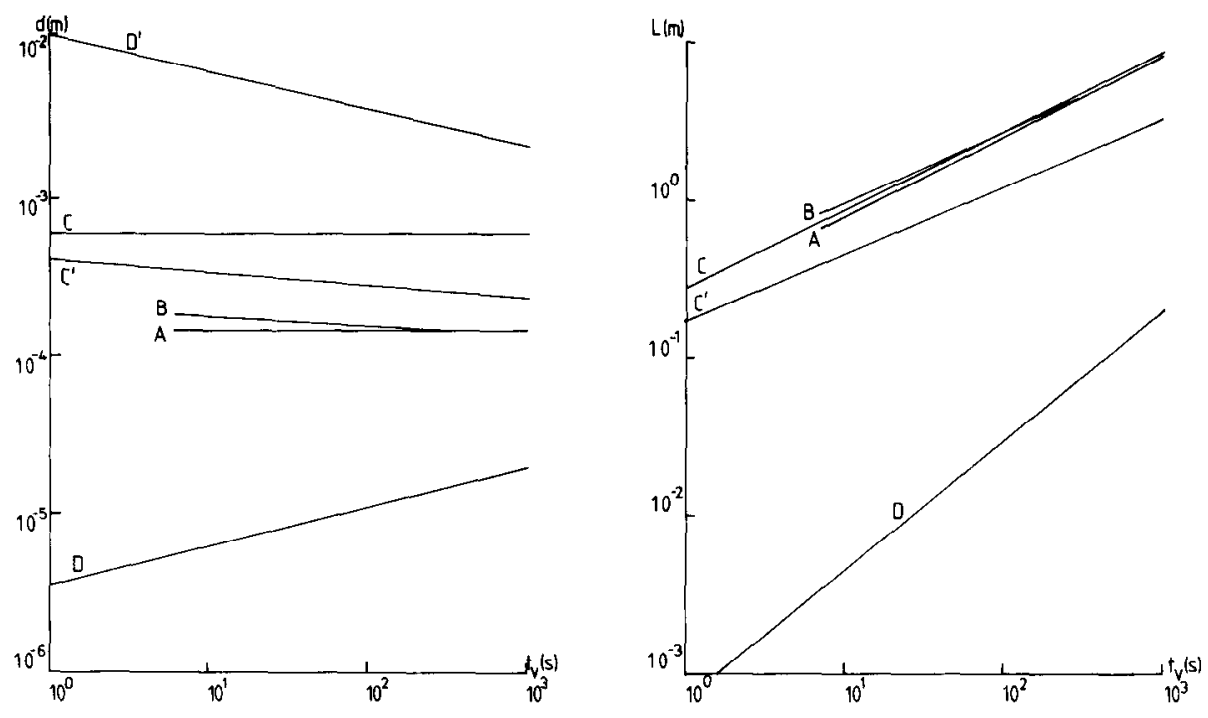

Fig. 2. Dimensions of the reactors as a function of the residence time $t_{v}$ Pressure drop, 1 bar. (A) Straight open tube; (B) coiled tube; (C') SBSR; all three reactors are operated at a constant reagent consumption of $8 \mu \mathrm{l}$. (D) Particle diameter for the packed reactors; $\left(D^{\prime}\right)$ bed diameter for the packed reactor operated at a constant reagent consumption of $8 \mu \mathrm{l}$.

Fig. 3. Reactor length as a function of the residence time $t_{\mathrm{v}}$ Pressure drop, 1 bar. (A) Straight open tube; (B) coiled tube; (C') SBSR; all three reactors are operated at a constant reagent consumption of $8 \mu$ l. (C) SBSR of a fixed geometry (see text); (D) packed reactor.

detector volume requirements. The SBSR with a constant geometry has the highest reagent consumption (case $\mathrm{C}$ ). This comparison enables several conclusions to be drawn. Thus, all four reactor types allow for sampling frequencies over $100 \mathrm{~h}^{-1}$ at residence times of about $100 \mathrm{~s}$ and at a reagent consumption of $8 \mu \mathrm{l}$ per analysis, at a pressure drop of 1 bar. The SBSR is characterized by a good performance at very favorable dimensions (e.g., design $\mathrm{C}$ ). It is a worthwhile alternative in f.i.a. for the packed reactor operated at optimal flow conditions.

The performance of the f.i.a. reactors may be compared with the AutoAnalyzer approach. Snyder [9] states that, for the SMAC analyzer operated at a flow rate of $600 \mu \mathrm{l} \mathrm{min}$ at a bubbling rate of $2 \mathrm{~s}^{-1}$, the sampling frequency is $265 \mathrm{~h}^{-1}$ at a residence time of $500 \mathrm{~s}$. This figure is higher than can be reached in f.i.a. with the present constraints, and the result is achieved at a reagent consumption of $150 \mu \mathrm{l}$ per analysis.

\section{Influence of the sample volume}

When the parameter $\alpha$ is defined as the ratio of the sample volume, $V_{\mathrm{s}}$ and the reactor volume, $V_{\boldsymbol{r}}$, it can be derived from earlier work [10] that for 

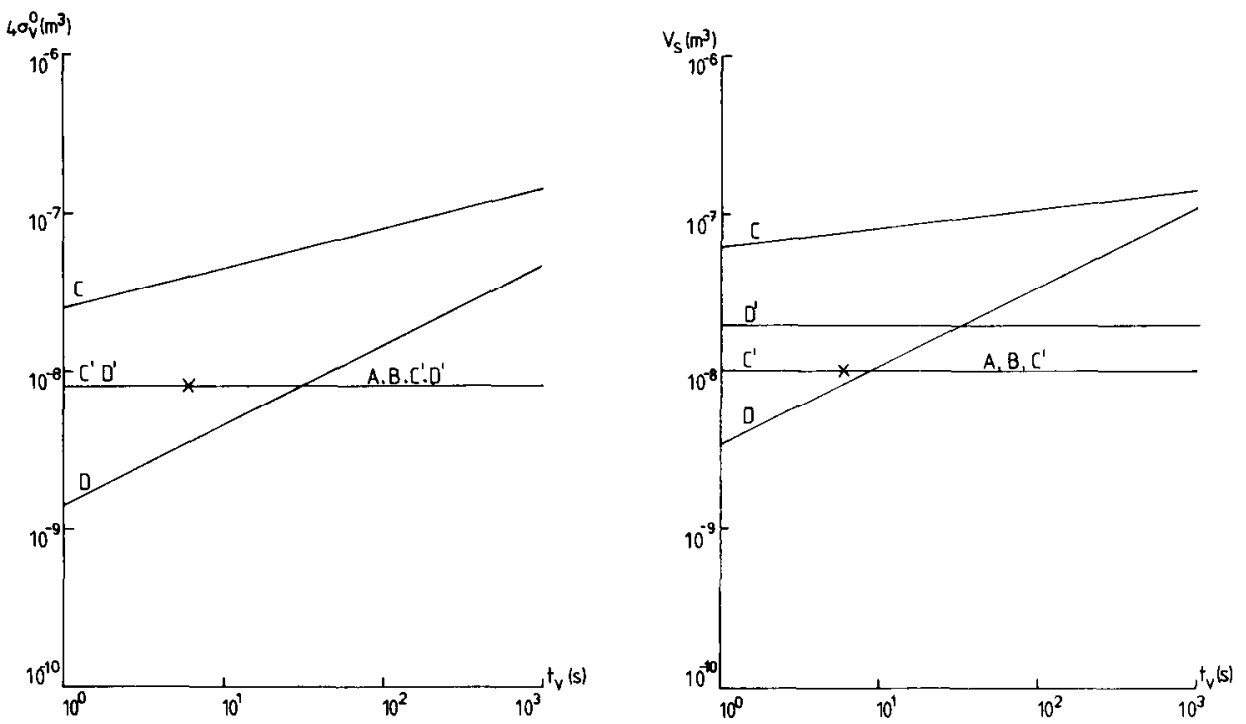

Fig. 4. Reagent consumption per analysis $\left(4 \sigma_{\mathrm{V}}^{0}\right)$ as a function of the residence time $t_{\mathrm{v}}$ Pressure drop, 1 bar. (A) Straight open tube; (B) coiled tube; (C') SBSR; (D') packed reactor; all reactors were designed with this variable as a constraint parameter. (C) SBSR with a constant geometry; (D) packed reactor with a constant bed diameter $(5 \mathrm{~mm})$.

Fig. 5. Maximum allowable sample volume as a function of the residence time $t_{\checkmark}$ Pressure drop, 1 bar. (A) Straight open tube; (B) coiled tube; (C') SBSR; all three reactors are operated at a reagent consumption of $8 \mu \mathrm{l}$. (D') Packed reactor with the same reagent consumption as the other three reactors; (C) SBSR of constant geometry; (D) packed reactor with a fixed bed diameter $(5 \mathrm{~mm})$.

a non-reacting sample a dilution factor of 2 is obtained when $\alpha n^{1 / 2}=2^{1 / 2}$. The time variance of the response curve in this case is $\sigma_{t}^{2}=\left(1 / n+\alpha^{2} / 12\right)$ $t_{\mathrm{v}}^{2}=(14 / 12 n) t_{\mathrm{v}}^{2}$. The sampling frequency is then given by

$f_{\max }=k_{1}\left(t_{\mathrm{v}}\right)^{-1} N^{1 / 2} 0.93$

It is seen that injection of a sample volume corresponding to $V_{s}=V_{r} 2^{1 / 2} /$ $n^{1 / 2}$ results in a slight loss of maximal sampling frequency, while the reactor is operated at a dilution of only two. The influence of chemical kinetics has been investigated [11]. It was concluded from that work that, for firstorder kinetics and adequate mixing of sample and reagent, it is useless to operate a f.i.a. reactor at residence times longer than needed to reach a conversion level of say $63 \%$ at sample volumes larger than corresponding to $\alpha n^{1 / 2}=2^{1 / 2}$. Quite straightforward calculations indicate that for the cases $A, B, C^{\prime}$ and $D^{\prime}$ the maximum allowable sample volume is independent of the residence time. Figure 5 illustrates the result of these calculations for $\alpha n^{1 / 2}=2^{1 / 2}$. The high sample volume that the SBSR can accommodate when operated at a constant particle diameter should be noted. 


\section{Conclusion}

Complete design equations for flow injection systems are presented; the equations allow optimization of these systems. The equations are evaluated for the optimization objectives of maximal sampling frequency and minimal reagent consumption. Constraints in the optimization procedure are the pressure drop which should not exceed 1 bar in f.i.a. and the detector volume. It is concluded that reduction of the detector volume is the most promising way of improving the performance of (miniaturized) f.i.a. systems.

The authors thank Dr. R. J. Jonker for valuable discussions and for his suggestion to apply the analysis of Knox and Saleem to f.i.a.

\section{REFERENCES}

1 J. Růżicka and E. H. Hansen, Flow Injection Analysis, Wiley, New York, 1981.

2 J. H. Knox and M. Saleem, J. Chromatogr. Sci., 7 (1969) 614.

3 J. H. Knox and M. T. Gilbert, J. Chromatogr., 186 (1979) 405.

4 G. Guiochon, Anal. Chem., 53 (1981) 1318.

5 J. M. Reijn, W. E. Van der Linden and H. Poppe, Anal. Chim. Acta, 126 (1981) 1.

6 H. Poppe, Anal. Chim. Acta, 114 (1980) 59.

7 R. Tijssen, Anal. Chim. Acta, 114 (1980) 71.

8 J. H. M. Van den Berg, R. S. Deelder and H. G. M. Egberink, Anal. Chim. Acta, 114 (1980) 91 .

9 L. R. Snyder, Anal. Chim. Acta, 114 (1980) 3.

10 J. M. Reijn, W. E. Van der Linden and H. Poppe, Anal. Chim. Acta, 114 (1980) 105.

11 J. M. Reijn, W. E. Van der Linden and H. Poppe, Anal. Chem., submitted. 